\title{
Comparison of Benign and Malignant Pilomatricomas Using Whole-exome Sequencing
}

\author{
MIN-KYUNG YEO and GO EUN BAE \\ Department of Pathology, Chungnam National University School of Medicine, Daejeon, Republic of Korea
}

\begin{abstract}
Background: Malignant pilomatricoma (MP) is a rare cancer of the hair matrix with only a few cases reported in literature. Given the rarity of this cancer and the lack of relevant genetic data, very little is known about the nature of the molecular pathophysiology except the involvement of the Catenin Beta 1 (CTNNB1)/Wnt/ $\beta$-catenin signaling pathway in some cases. Materials and Methods: We describe the whole-exome genomic profiling of four samples from two patients: 1) an MP from patient I, 2) a coexisting benign pilomatricoma $(B P)$ from patient $I, 3)$ a BP from an age and location-matched control patient II, and 4) normal skin tissue from patient II. Results: We detected a pathogenic somatic missense mutation in fibroblast growth factor receptor 4 (FGFR4) (c.1162G>A, p. Gly388Arg) in MP and coexisting $B P$ in patient $I$, whereas the control BP harbored the classical CTNNB1 mutant. Conclusion: This study, the first comparative analysis of benign and MP through whole-exome analysis, identified a novel oncogenic mutation in FGFR4.
\end{abstract}

Malignant pilomatricomas (MPs) are extremely rare adnexal cancers originating from hair matrix cells. Fewer than 150 cases of MPs have been reported in literature worldwide and the vast majority as case reports (1-4). MP has significant recurrence and metastatic potential, but due to its slow growth and rarity, it is often clinically misdiagnosed as a sebaceous cyst or other common skin tumor, potentially delaying tumor resection (4). MP can arise de novo as a solitary lesion or through the transformation of a benign pilomatricoma (BP); however, no available immunohistochemical or molecular methods are capable of distinguishing BP from MP.

This article is freely accessible online.

Correspondence to: Go Eun Bae MD, Ph.D., Chungnam National University School of Medicine, Munwha-ro 266, Jung-gu, Daejeon, 35015, Republic of Korea. Tel: +82 422807797, Fax: +82 422807189, e-mail: goeunbae1@gmail.com

Key Words: CTNNB1, FGFR4, malignant, mutation, pilomatricoma, whole-exome sequencing.
Consequently, the diagnosis of malignancy must be based on histological examination (1). To date, the CTNNB1/Wnt/ $\beta$ catenin signaling pathway, identified through selective molecular analysis, is the only tumorigenic factor shown to drive pilomatricoma $(3,5)$.

Here, we report the first case of MP subjected to wholeexome and mutational signature analysis. To obtain insight into the molecular mechanisms that might be responsible for malignant transformation, we compared the detailed molecular and immunologic characteristics among four samples from two patients: 1) malignant pilomatricoma (patient I), 2) coexisting benign pilomatricoma (patient I), 3) benign pilomatricoma (patient II), and 4) normal skin tissue (patient II). All cases were subjected to whole-exome sequencing (WES), mutational signature (MS) analysis, Sanger sequencing, and immunohistochemical studies.

\section{Materials and Methods}

Patients and tissue samples. The MP sample, from patient I, was identified by retrospectively searching the pathology database at Chungnam National University Hospital, Daejeon, South Korea, with approval from the hospital's Institutional Review Board (202007-063). An age- and site-matched benign pilomatricoma (patient II) was selected as a control for this study, also from the case files of Chungnam National University. All available slides were reviewed by two licensed pathologists (Bae and Yeo). Clinical features and follow-up were obtained by review of the patients' electronic medical records. This study (2020-07-063) was reviewed and approved by the Institutional Review Board at the Chungnam National University Hospital, Daejeon, Republic of Korea.

Whole-exome sequencing and data analysis. Three benign and malignant pilomatricomas and one control normal skin sample underwent WES, performed as an outsourced service by Macrogen (Seoul, Republic of Korea). DNA was extracted from formalinfixed, paraffin-embedded (FFPE) tissue sections. High-quality DNA (200 ng) was subjected to library preparation and exome capture using the SureSelect Target Enrichment System for Illumina PairedEnd Multiplexed Sequencing Library (Illumina, San Diego, CA, USA). Captured material was indexed and sequenced on the Illumina HiSeq2500 platform, yielding an average sequence coverage of $>40 \times$. All procedures were performed according to the manufacturer's instructions as reported in the literature $(6,7)$. The 

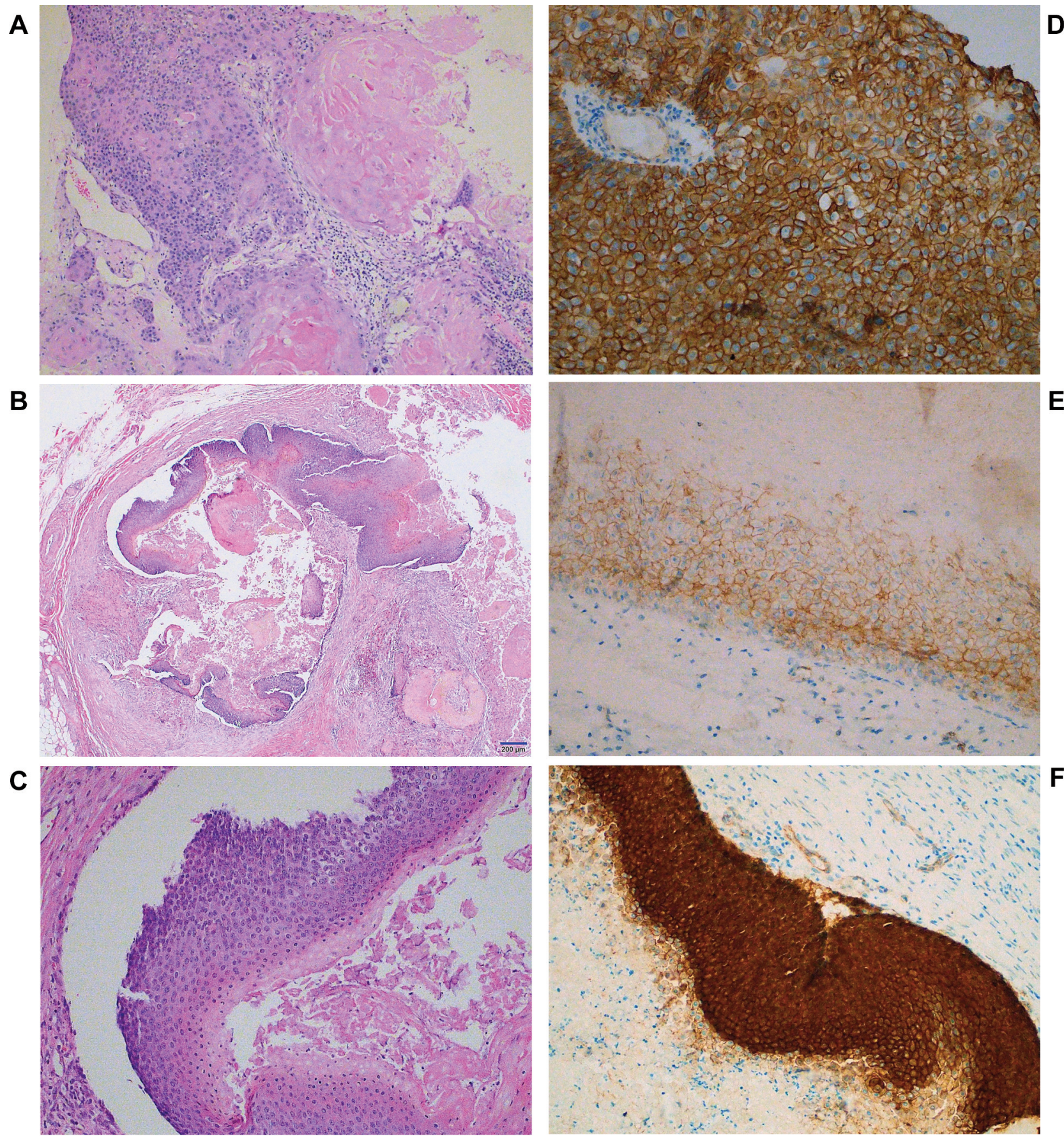

Figure 1. Continued

resultant FASTQ files were aligned to the National Center for Biotechnology Information (NCBI) build 37.2 of the human reference genome (hg19) with default paired-end parameters. Quality metrics were calculated using the MuTeck2 software. (v4.1.0.0, CA, USA), and sequence data were arranged to mark duplicate reads and recalibrate quality scores, and were realigned around known polymorphisms using the Genome Analysis Toolkit
(GATK v3.8, CA, USA) (8). The extracted data were further interpreted to standardize pathogenic clinical significance according to ClinVar-indexed variants (NCBI, USA) (9). A variant was considered positive if pathogenic or likely pathogenic variants corresponded to the phenotype and the mode of inheritance $(10,11)$. Sanger sequencing. The MP underwent direct sequencing as an outsourced service by Macrogen (Seoul, Republic of Korea). DNA 


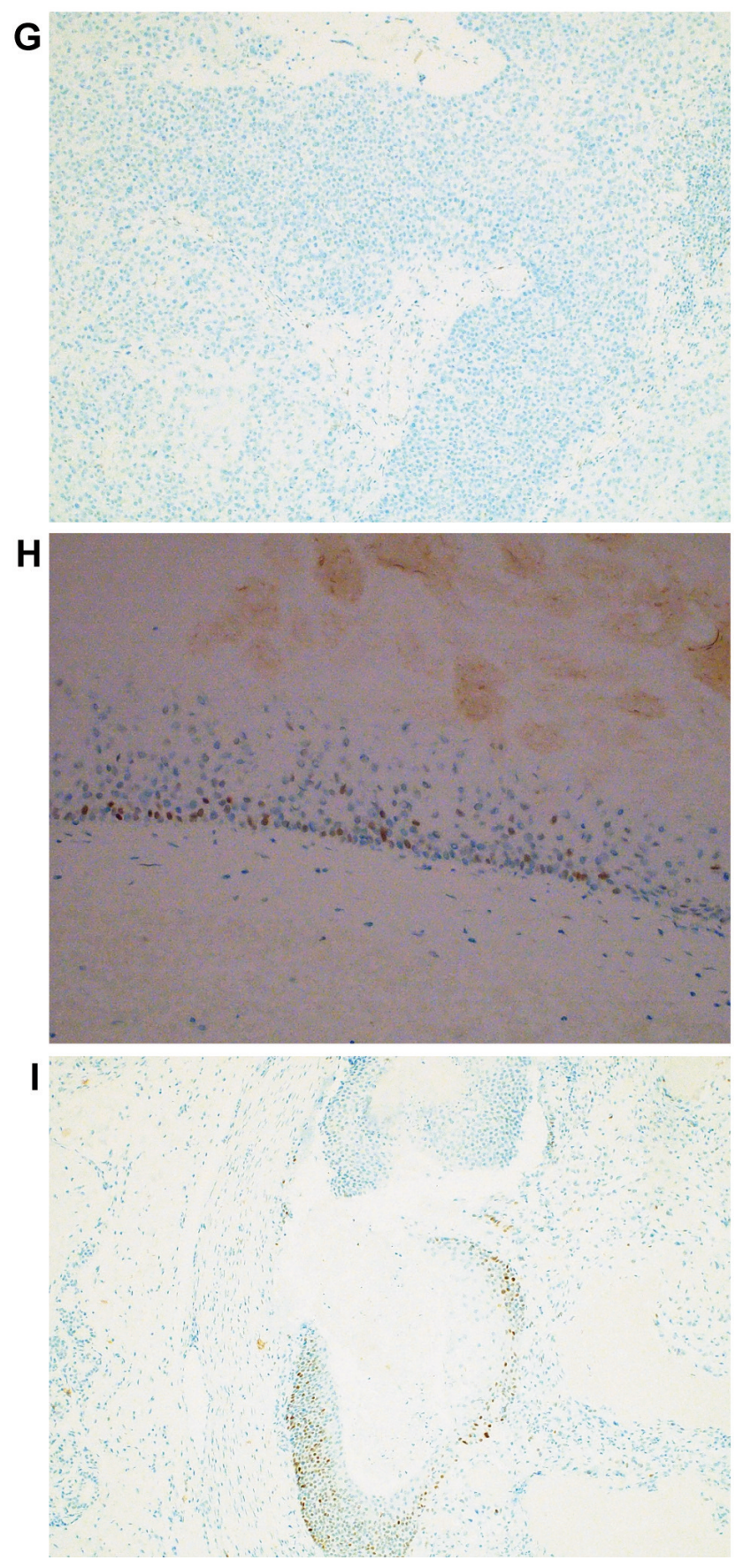

Figure 1. Pathologic and Immunohistochemical (IHC) features of benign and malignant pilomatricomas. (A) Malignant pilomatricoma $(M P)$ presents basaloid cell nests with stromal invasion and immature keratinization [100x, Hematoxylin-eosin stain (HE)]. BP from patient $I(B)$ and control patient II $(C)$ reveal keratinizing basaloid cell nests without cytological atypia. $(D-F)$ Expression patterns of $\beta$-catenin IHC differ between two patients; membranous staining in patient $I$ $[M P(D) B P(E)] v s$. strong nuclear and cytoplasmic expression in $B P$ from control patient II $(F, 200 x)$. p53 expression patterns differed between benign and malignant tumors (G-I: negative (null) staining in $M P(G)$ vs. wild-type expression in both BPs (H from patient $I, I$ from control patient II). was extracted from an FFPE block from the MP and quantified by QIAxpert (QIAGEN, Hilden, Germany) analysis, yielding a concentration of $28.4 \mathrm{ng} / \mu \mathrm{l}$. A fragment of the human FGFR4 exon 9 was amplified with PCR products that were cloned into plasmids and sequenced using the $\operatorname{BigDye}(\mathrm{R})$ Terminator v3.1 Cycle Sequencing Kit (CE Sequencing Instruments, San Jose, CA, USA). Sequence identification and variant analysis were performed using Variant Reporter 2.1 (Applied Biosystems, CA, USA) and DNASTAR Lasergene SeqMan 7.0 (WI, USA). For all analyses, data obtained with the forward and reverse primers were combined and aligned to the consensus sequence obtained from the GenBank database (NCBI Reference Sequence: NG_012067.1).

Mutational signature analysis. To obtain additional clues about the causative origins of cancer, extracted somatic point mutations were mapped to the 96 possible trinucleotide contexts for signature analysis (12). To reconstruct the mutation profile of each sample, the data were then analyzed with DeconstructSigs 1.9, a multiple linear regressionbased algorithm, using a linear combination predefined by the Signatures of Mutational Processes in Human Cancer database found at http://cancer.sanger.ac.uk/cosmic/signatures (12).

Immunohistochemical studies. FFPE tissue blocks of four samples from two patients (patient I: MP and coexisting BP; patient II: BP and normal skin tissue) were sectioned at $4 \mu \mathrm{m}$ thickness. Sections were deparaffinized in xylene and rehydrated through a graded alcohol series. Immunohistochemical staining (IHC) was performed using an automatic immunostaining instrument (Ventana Benchmark XT; Ventana Medical Systems, Tucson, AZ, USA). Commercially available antibodies were used including $\beta$-catenin (Invitrogen, Carlsbad, California, USA; 1:500 dilution) and p53 (DAKO, Glostrup, Denmark; 1:300 dilution).

\section{Results}

\section{Clinicopathologic characteristics}

Patient history. A 43-year-old woman with no significant past medical history presented with a palpable nodule under the superior scalp that had been present for a few years. Upon a radiologic CT scan, the nodules exhibited a well-confined subcutaneous cystic lesion without peripheral soft tissue invasion. The radiologist suggested BP and epidermal cyst as differential diagnoses. Excisional biopsy of the lesion revealed a well-circumscribed, dome-shaped, whitish cystic tumor containing necrotic inner contents, measuring $2.1 \mathrm{~cm}$ in maximum diameter, mainly located in the dermis and subcutaneous tissue of the excised skin. The diagnosis of MP was supported by the presence of focal pleomorphic atypical cell nests with multiple abnormal mitosis (Figure 1A) admixed with the BP (Figure 1B, BP1), as determined by microscopy. The findings suggested malignant transformation within the coexisting BP. The pathology of age- and site-matched benign control pilomatricoma (BP2) is shown in Figure 1C.

Immunohistochemical findings. The IHC expression patterns of the $\beta$-catenin from the two patients are distinct: membranous expression in MP (Figure 1D) and coexisting BP1 (Figure 1E) from patient I vs. strong nuclear and cytoplasmic $\beta$-catenin 
expression in BP2 from the control patient (Figure 1F). By contrast, the patterns of p53 expression differ between the benign and malignant tumors: completely negative expression in MP (Figure 1G), indicative of functional loss due to TP53 mutation, vs. wild-type p53 expression in both BPs from the two patients (Figure 1H, I). In addition, we performed IHC studies to screen for abnormalities in DNA mismatch repair proteins, including MLH1, MSH2, PMS2, and MSH6; in all cases, the results revealed no microsatellite instability (data not shown).

\section{Molecular analysis}

Somatic variant profiling and mutational classification. Three pilomatricomas and normal control skin tissue were sequenced and analyzed by WES. The average numbers of reads for tumor and normal samples were 256,668,105 and 114,853,678, respectively, generating an average coverage of $96.1 \times$ and $99 \times$ across $99 \%$ of the captured sequence. The average number of somatic missense mutations identified in exomes ranged from 11.439 to 11.623 (Figure 2A). Overall, the extracted data revealed that the types and amounts of somatic variants were similar between the two benign and one malignant pilomatricomas (Figure 2A). However, after filtering by mutational classification, only the FGFR4 (c.1162G>A, p. Gly388Arg, NM 213647.3) missense mutation remained as a deleterious pathogenic mutation in both MP and the coexisting BP1 (Figure 2B). In contrast to previous studies, CTNNB1 (c.98C >T, p. Ser33Phe, NM001904.4) was identified as a pathogenic mutation only in the control BP2 (patient II) (Figure 2B). We further examined the extracted data, focusing on somatic variants that have been suggested by previous studies to be potentially targetable genetic mutants in hair matrix and adnexal cancers including TP53, HRAS, RB1, ATM, ARIDIA, APC PTEN, EGFR, CDKN2A, CDKN2B, CDKN1A, ALPK1, DNMT3A, PIK3CA, CYLD, NOTCH1, NOTCH2, ZNF750, RREB1, KMT2D, KRAS, PTCH1, TCF7L1, MLH1, MSH2, MSH6, PMS2, POLE, and FAT3. No significant pathogenic genomic alterations were identified among these genes by mutational classification (14-21). An additional splice-donor and intron variant in TP53 (c.375+1G>A, NM 000546.5) was only present in the MP (Figure $2 \mathrm{~B}$ ).

Sanger sequencing. Exon 9 of FGFR4 was additionally analyzed by Sanger (direct) sequencing from the remaining tumor tissue from MP and BP1. FGFR4 mutation was confirmed at codon 388 (Gly388Arg) in both the forward and reverse sequences as a heterozygous single-nucleotide polymorphism (Figure 2C, red arrow).

Mutational signature analysis. Computational analysis of the MS allowed us to attribute mutations to environmental exposure and mutagens (22-24). The MP and coexisting BP1 shared most MS patterns, including single base substitution
(SBS) 1, SBS12, SBS16, SBS32, and SBS39, whereas the control patient's BP had a different MS configuration, except for SBS1 (Figure 3A). The patterns and composition from the MP and coexisting BP1 were similar but had no predominant MS type, whereas the control BP2 had different MS patterns with predominance of SBS1 (>60\%). The 96 mutational classes of all SNVs within the three tumors shared the main mutational processes: $\mathrm{C}>\mathrm{T}$ changes represented SBS1, whereas minor $\mathrm{T}>\mathrm{C}$ changes were identified as SBS12, 16, and 32 in tumors, except for the control tumor BP2 (Figure 3B).

\section{Discussion}

Malignant pilomatricoma (MP), a very rare adnexal cancer with a high rate of local recurrence but uncommon distant metastasis, has not been extensively characterized at the molecular level. To date, CTNNB1, the gene encoding $\beta$ catenin, is the only driver mutation identified in both benign and MPs (3, 13). Magdalena et al. as well as Katharina et al. have reported a few pediatric cases of multiple pilomatricoma with CTNNB1 mutations, associated with germline mutations in mismatch repair genes $(14,15)$. The reported frequency of the CTNNB1 mutation in pilomatrical tumor varies from $30 \%$ to $100 \%(16,17)$, raising the question of whether actionable mutations in pilomatrical tumors occur in genes other than CTNNB1.

In this study, we identified a novel FGFR 4 c. $1162 \mathrm{G}>\mathrm{A}$ missense mutation in MP and its coexisting BP through WES. FGFR4 is normally highly expressed in embryonic tissues and is involved in embryonic development, angiogenesis, and tissue differentiation, whereas in adult tissues its expression is limited (18). FGFR4 mutations activate oncogenic signaling pathways and have been observed in breast, colorectal, lung, and melanocytic skin cancers (29-31). The c.1162G $>$ A mutation, which is predominant in these contexts, changes the encoded amino acid residue (codon 388 in exon 9, located in the transmembrane domain) from glycine to arginine, exposing a membrane-proximal signal transducer and activator of transcription 3 (STAT3) binding site that activates the downstream STAT3 signaling cascade $(19,20)$.

In several cancers, $\operatorname{Arg} 388$ allele carriers have significantly poorer prognoses than homozygous Gly388 allele carriers (20). FGFR4 Gly388Arg mutants are present in $55 \%$ of skin melanoma patients and $38 \%$ of patientderived melanoma cell lines, and are correlated with poor tumor thickness and invasiveness in these cancers (21-23). The frequency of FGFR4 mutation has not been evaluated in non-melanocytic skin cancer; hence, further studies are needed to assess the prognostic risks associated with FGFR4 mutations in skin adnexal tumors, including pilomatrical ones (2). 
A

45,000
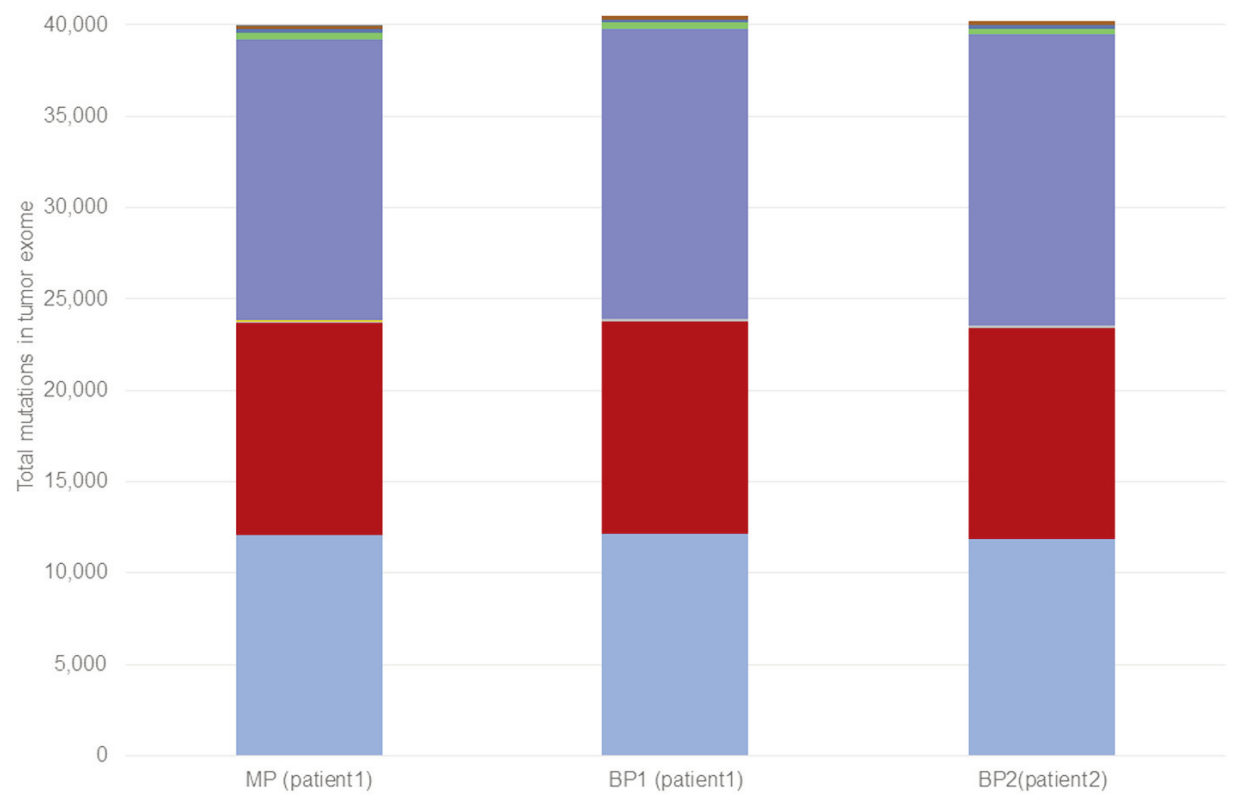

- Inframe Deletion

- Inframe Insertion

a Frameshift Variant

- Indels

- Stop Lost

- Stop Gained

- Missense Variant

- Synonymous SNPS

B

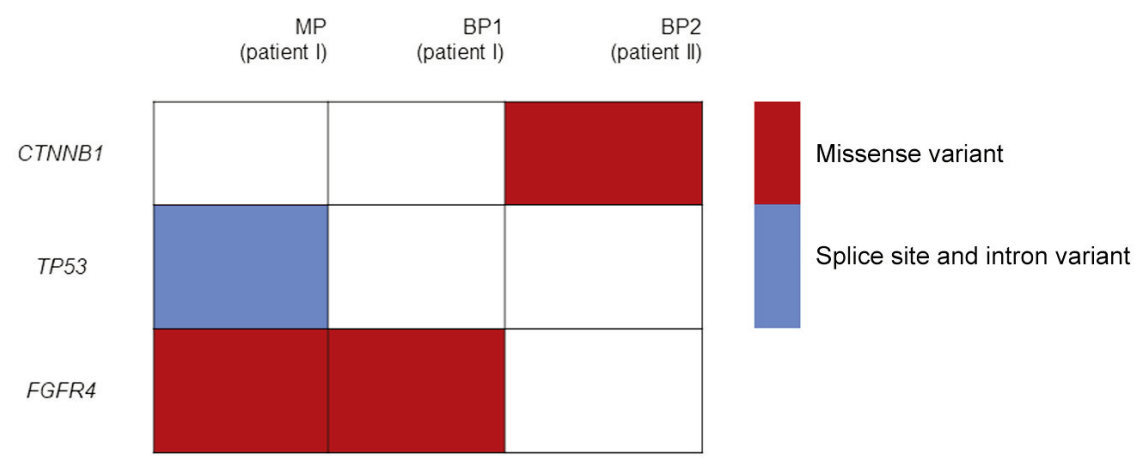

C
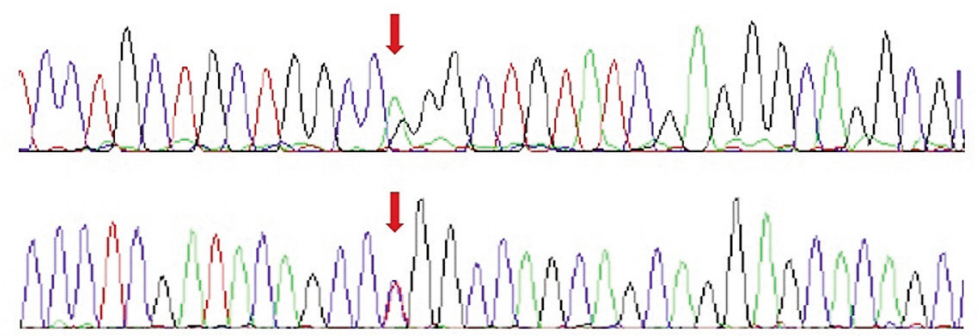

Figure 2. Molecular profiling of pilomatricomas. (A) Overall amounts of somatic mutation and variant types were similar among all tumors. (B) After filtering by mutational classification, the pathogenic variants differed: FGFR4 in malignant pilomatricoma (MP) vs. benign pilomatricoma (BP1) from patient I, and CTNNB1 in control BP2. The TP53 splicing donor and intron variant were identified only in MP. (C) Chromatogram of exon 9 from FGFR4 gene obtained by Sanger sequencing with forward (upper) and reverse (lower) primer. The arrow indicates a clear heterozygous double peak (red arrow) at c.1198, generating the G388A missense mutation. 

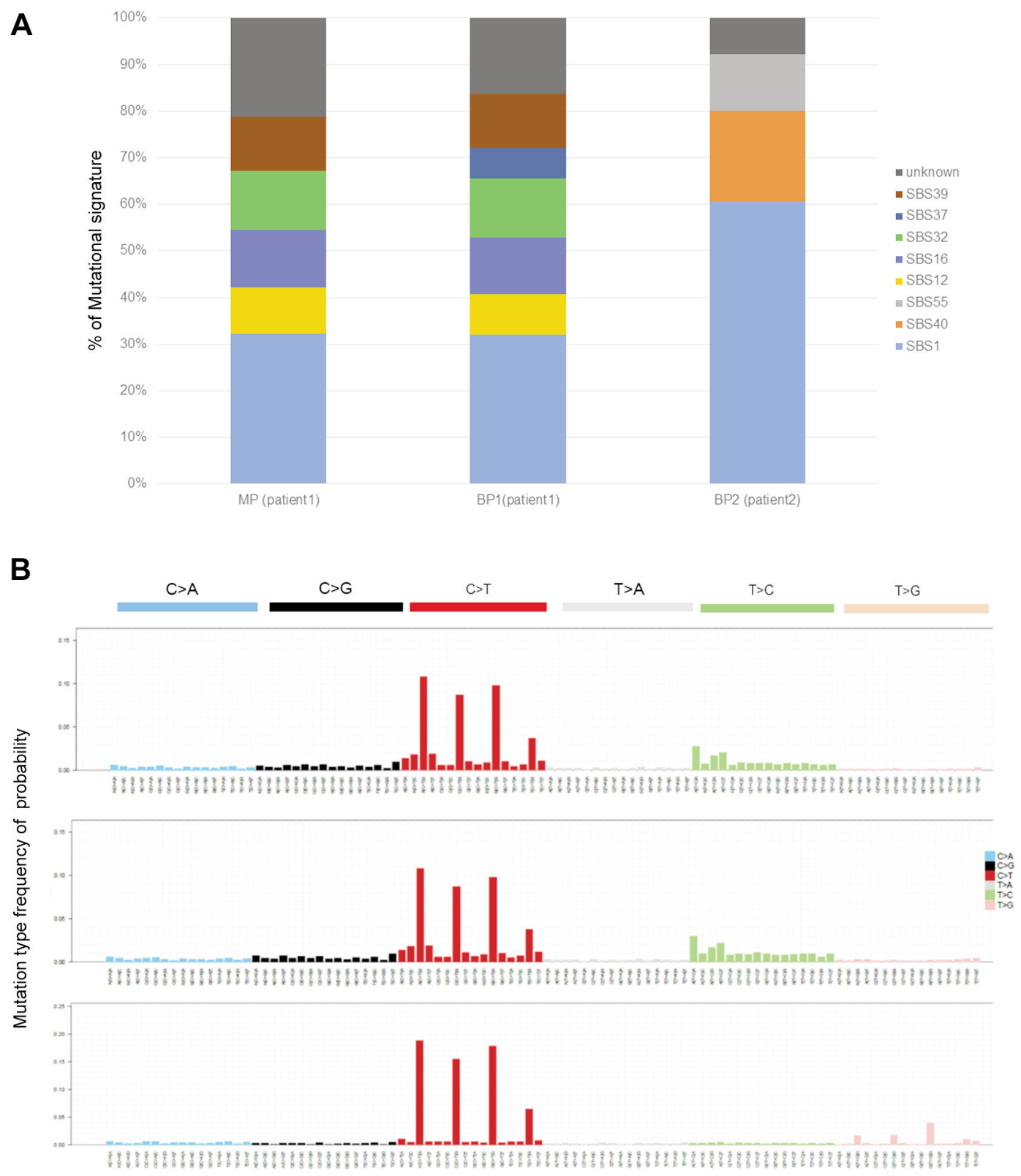

Figure 3. Mutational spectra and signature analysis. (A) Bars show various mutational signatures (MS) extracted in pilomatrical tumors. Patterns and composition from MP and coexisting BP1 are similar but exhibit no predominant MS type, whereas control BP2 exhibit different MS patterns with predominant single base substitution (SBS) 1 (>60\%). (B) The 96 mutational classes of all SNVs; no predominant sequence context was identified.

The molecular pathogenesis of skin cancer remains under investigation, but UV radiation-induced damage is one of the most frequent oncogenic drivers causing impairment of immune suppression in skin cancers, which have the highest mutational burden $(2,24)$. Because pilomatrical tumors frequently occur in sun-exposed areas such as the head and neck, previous studies suggested that UV radiation may contribute to malignant transformation (2). Somatic mutations in cancer genomes are caused by multiple mutational processes, each of which generates unique combinations of mutation types termed "mutational signatures," which provide a powerful tool for identifying the cancer-causing agents (25). UV MSs, defined as a preponderance of $\mathrm{C} \rightarrow \mathrm{T}$ substitutions at dipyrimidine sites, including $\mathrm{CC} \rightarrow \mathrm{TT}$, have been detected in the TP53 tumor suppressor gene from squamous cell carcinoma (SCC), basal 
cell carcinoma, precursors of SCC, and even normal sunexposed skin $(26,27)$. A generally confirmed UV signature is defined as follows: $\geq 60 \%$ of mutations are $\mathrm{C} \rightarrow \mathrm{T}$ at dipyrimidine sites, with $\geq 5 \% \mathrm{CC} \rightarrow \mathrm{TT}$ (27). The extracted MS data from pilomatrical tumors, however, showed $<30 \%$ $\mathrm{C} \rightarrow \mathrm{T}$ changes (mostly associated with SBS1), with no predominant MS patterns, precluding UV radiation as a tumorigenic factor. SBS1 was the most prominent pattern in all pilomatrical tumors (Figure 2A). SBS1 mutations, associated with deamination of 5-methyl cytosine, are correlated with age and arise at different rates in different tissue types (28). Although SBS1 has not been implicated in specific causes of cancer, the distinct MS configuration in MP and BP1 with the FGFR4 mutations suggests the existence of alterations in oncogenic pathways other than CTNNB1/Wnt/ $\beta$-catenin signaling.

Loss of function in TP53 plays a role in the acquisition of new genetic events contributing to malignant transformation in many cancers. In contrast to benign tumors, we observed complete loss of p53 only within MP with the TP53 splicing variant; thus, inactivation of the p53 tumor suppressor is considered to be crucial in the final step of carcinogenesis. Currently, because we have no molecular methods for distinguishing MP from BP, p53 IHC could be useful in the diagnosis of malignancy. $\beta$-catenin IHC patterns also reflect innate CTNNB1 status: in this study, nuclear and cytoplasmic $\beta$-catenin immunostaining corresponded to CTNNB1 mutations in the control benign tumor, whereas normal membranous expression was detected in both MP and BP1, which harbored the oncogenic mutation in FGFR4. IHC studies could be very useful for predicting the underlying oncogenic pathways in pilomatrical tumors.

In summary, we have reported a novel FGFR4 mutant and illustrated MS patterns in pilomatrical tumors. The results suggest the existence of a distinct tumorigenic pathway other than $C T N N B 1 / \mathrm{Wnt} / \beta$-catenin signaling in these tumors. This is the first extensive genetic analysis of MP and BP through WES and MS analysis. Given the rarity of pilomatricomas and the lack of relevant genetic data, further work is necessary to confirm the incidence of this mutation and its clinical significance.

\section{Conflicts of Interest}

No potential conflicts of interest are disclosed by the authors. The funders had no role in the design of the study or in the decision to publish the results.

\section{Authors' Contributions}

Bae and Yeo had full access to all of the data in the study and take responsibility for the integrity of the data and the accuracy of the data analysis. Study concept and design: Yeo; Acquisition, analysis, and interpretation of data: Bae; Drafting of the manuscript: Bae;
Critical revision of the manuscript for important intellectual content: Bae and Yeo; Obtained funding: Bae and Yeo; Administrative technical, or material support: Yeo; Study supervision: Bae and Yeo.

\section{Acknowledgements}

The biospecimens and data used for this study were provided by the Biobank of Chungnam National University Hospital, a member of the Korea Biobank Network.

\section{Funding}

This research was supported by the National Research Foundation of Korea (NRF) grant funded by the Korea government (MSIT) (2019R1G1A1100578), and the Bio \& Medical Technology Development Program of the National Research Foundation (NRF) \& funded by the Korean government (MSIT) (2019M3E5D1A0 2068558).

\section{References}

1 Melancon JM, Tom WL, Lee RA, Jackson M and Jiang SI: Management of pilomatrix carcinoma: a case report of successful treatment with Mohs micrographic surgery and review of the literature. Dermatol Surg 37: 1798-1805, 2011. PMID: 22093235. DOI: 10.1111/j.1524-4725.2011.02170.x

2 Parra L, Martin M, Garrido M, Pedraza JM, Palazon J, Robustillo M, Grandes D and Lagaron E: Concurrent pilomatrix carcinoma and diffuse large B-cell lymphoma. Oncol Ther 4: 129-134, 2016. PMID: 28261645. DOI: 10.1007/s40487-0160021-3

3 Lazar AJ, Calonje E, Grayson W, Dei Tos AP, Mihm MC, Jr. and Redston M: Pilomatrix carcinomas contain mutations in CTNNB1, the gene encoding beta-catenin. J Cutan Pathol 32: 148-157, 2005. PMID: 15606674. DOI: 10.1111/j.03036987.2005.00267.x

4 Herrmann JL, Allan A, Trapp KM and Morgan MB: Pilomatrix carcinoma: 13 new cases and review of the literature with emphasis on predictors of metastasis. J Am Acad Dermatol 71: 38-43 e2, 2014. PMID: 24739254. DOI: 10.1016/j.jaad.2014.02.042

5 Kazakov DV, Sima R, Vanecek T, Kutzner H, Palmedo G and Kacerovska D: Mutations in exon 3 of the CTNNB1 gene (betacatenin gene) in cutaneous adnexal tumors. Am J Dermatopathol 31: 248-255, 2009.

6 Kozarewa I and Turner DJ: Amplification-free library preparation for paired-end Illumina sequencing. Methods Mol Biol 733: 257-266, 2011. PMID: 19384065. DOI: 10.1097/DAD.0b013e318198922a

7 Margraf RL, Durtschi JD, Dames S, Pattison DC, Stephens JE and Voelkerding KV: Variant identification in multi-sample pools by illumina genome analyzer sequencing. J Biomol Tech 22: 7484, 2011. PMID: 21738440.

8 Van der Auwera GA, Carneiro MO, Hartl C, Poplin R, Del Angel $\mathrm{G}$ and Levy-Moonshine A: From FastQ data to high confidence variant calls: the Genome Analysis Toolkit best practices pipeline. Curr Protoc Bioinformatics 43: 11.10.1-11.10.33, 2013. PMID: 25431634. DOI: 10.1002/0471250953.bi1110s43

9 Landrum MJ, Lee JM, Benson M, Brown G, Chao C and Chitipiralla S: ClinVar: public archive of interpretations of 
clinically relevant variants. Nucleic Acids Res 44: D862-86, 2016. PMID: 26582918. DOI: 10.1093/nar/gkv1222

10 Richards S, Aziz N, Bale S, Bick D, Das S and Gastier-Foster J: Standards and guidelines for the interpretation of sequence variants: a joint consensus recommendation of the American College of Medical Genetics and Genomics and the Association for Molecular Pathology. Genet Med 17: 405-424, 2015. PMID: 25741868. DOI: $10.1038 /$ gim.2015.30

11 Li MM, Datto M, Duncavage EJ, Kulkarni S, Lindeman NI and Roy S: Standards and guidelines for the interpretation and reporting of sequence variants in cancer: A joint consensus recommendation of the Association for Molecular Pathology, American Society of Clinical Oncology, and College of American Pathologists. J Mol Diagn 19: 4-23, 2017. PMID: 27993330. DOI: 10.1016/j.jmoldx.2016.10.002

12 Alexandrov LB, Nik-Zainal S, Wedge DC, Aparicio SA, Behjati $\mathrm{S}$ and Biankin AV: Signatures of mutational processes in human cancer. Nature 500: 415-421, 2013. PMID: 23945592. DOI: 10.1038 /nature 12477

13 Rubben A, Wahl RU, Eggermann T, Dahl E, Ortiz-Bruchle N and Cacchi C: Mutation analysis of multiple pilomatricomas in a patient with myotonic dystrophy type 1 suggests a DM1associated hypermutation phenotype. PLoS One 15: e0230003, 2020. PMID: 32155193. DOI: 10.1371/journal.pone.0230003

14 Chmara M, Wernstedt A, Wasag B, Peeters H and Renard M and Beert E: Multiple pilomatricomas with somatic CTNNB1 mutations in children with constitutive mismatch repair deficiency. Genes Chromosomes Cancer 52: 656-664, 2013. PMID: 23629955. DOI: $10.1002 / \mathrm{gcc} .22061$

15 Wimmer K, Beilken A, Nustede R, Ripperger T, Lamottke B and Ure B: A novel germline POLE mutation causes an early onset cancer prone syndrome mimicking constitutional mismatch repair deficiency. Fam Cancer 16: 67-71, 2017. PMID: 27573199. DOI: 10.1007/s10689-016-9925-1

16 Kajino Y, Yamaguchi A, Hashimoto N, Matsuura A, Sato N and Kikuchi K: beta-Catenin gene mutation in human hair folliclerelated tumors. Pathol Int 51: 543-548, 2001. PMID: 11472567. DOI: 10.1046/j.1440-1827.2001.01231.x

17 Ha SJ, Kim JS, Seo EJ, Lee KH, Lee HJ and Kim JW: Low frequency of beta-catenin gene mutations in pilomatricoma. Acta Derm Venereol 82: 428-431, 2002. PMID: 12575848. DOI: 10.1080/000155502762064557

18 Thisse B and Thisse C: Functions and regulations of fibroblast growth factor signaling during embryonic development. Dev Biol 287: 390-402, 2005. PMID: 16216232. DOI: 10.1016 /j.ydbio.2005.09.011
19 Marme F, Hielscher T, Hug S, Bondong S, Zeillinger R and Castillo-Tong DC: Fibroblast growth factor receptor 4 gene (FGFR4) 388Arg allele predicts prolonged survival and platinum sensitivity in advanced ovarian cancer. Int J Cancer 131: E586591, 2012. PMID: 22034009. DOI: 10.1002/ijc.27329

20 Tang S, Hao Y, Yuan Y, Liu R and Chen Q: Role of fibroblast growth factor receptor 4 in cancer. Cancer Sci 109: 3024-3031, 2018. PMID: 30070748. DOI: 10.1111/cas.13759

21 Streit S, Mestel DS, Schmidt M, Ullrich A and Berking C: FGFR4 Arg388 allele correlates with tumour thickness and FGFR4 protein expression with survival of melanoma patients. Br J Cancer 94: 1879-1886, 2006. PMID: 16721364. DOI: 10.1038/sj.bjc.6603181

22 Hartman ML, Sztiller-Sikorska M and Czyz M: Whole-exome sequencing reveals novel genetic variants associated with diverse phenotypes of melanoma cells. Mol Carcinog 58: 588-602, 2019. PMID: 30556601. DOI: 10.1002/mc.22953

23 Bange J, Prechtl D, Cheburkin Y, Specht K, Harbeck N and Schmitt M: Cancer progression and tumor cell motility are associated with the FGFR4 Arg(388) allele. Cancer Res 62: 840847, 2002. PMID: 11830541.

24 Narayanan DL, Saladi RN and Fox JL: Ultraviolet radiation and skin cancer. Int J Dermatol 49: 978-986, 2010. PMID: 20883261. DOI: 10.1111/j.1365-4632.2010.04474.x

25 Phillips DH: Mutational spectra and mutational signatures: Insights into cancer aetiology and mechanisms of DNA damage and repair. DNA Repair (Amst) 71: 6-11, 2018. PMID: 30236628. DOI: 10.1016/j.dnarep.2018.08.003

26 Brash DE, Rudolph JA, Simon JA, Lin A, McKenna GJ and Baden HP: A role for sunlight in skin cancer: UV-induced p53 mutations in squamous cell carcinoma. Proc Natl Acad Sci USA 88: 10124-10128, 1991. PMID: 1946433. DOI: 10.1073/ pnas.88.22.10124

27 Brash DE: UV signature mutations. Photochem Photobiol 91: 15-26, 2015. PMID: 1946433. DOI: 10.1073/pnas.88.22.10124

28 Alexandrov LB, Kim J, Haradhvala NJ, Huang MN, Tian Ng $\mathrm{AW}$ and $\mathrm{Wu} \mathrm{Y}$ : The repertoire of mutational signatures in human cancer. Nature 578: 94-101, 2020. PMID: 32025018. DOI: $10.1038 / \mathrm{s} 41586-020-1943-3$
Received August 7, 2020

Revised September 2, 2020 Accepted September 7, 2020 